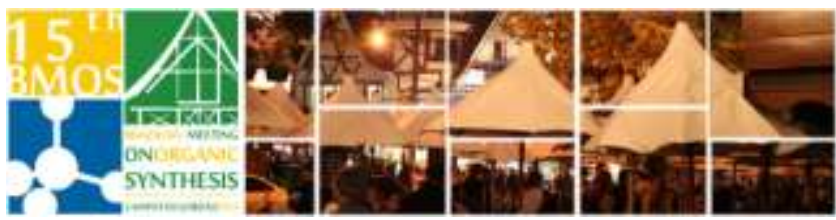

\title{
Synthesis of lactones and lactams analogues to rubrolides as inhibitors of Enterococcus faecalis biofilm formation
}

\author{
Ulisses A. Pereira ${ }^{a}$, Luiz C. A. Barbosa ${ }^{b_{*}}$, Célia R. A. Maltha ${ }^{a}$, Antônio Jacinto \\ Demuner ${ }^{\mathrm{a}}$, Andréa de L. Pimenta ${ }^{\mathrm{c}, \mathrm{d}}$
}

a'Department of Chemistry, Federal University of Viçosa, Av. PH Rolfs, Viçosa, MG, 36570-000,

${ }^{b}$ Department of Chemistry, Federal University of Minas Gerais, Av. Pres. Antônio Carlos, 6627,

Campus Pampulha, CEP 31270-901, Belo Horizonte, MG, Brazil/ ${ }^{C}$ Department of Biologie, ERRMECe, University of Cergy Pontoise, 2, Av. Adolphe Chauvin 95302 Cergy Pontoise - France

${ }^{d}$ Department of Odontology, Federal University of Santa Catarina 88040-900, Florianópolis, SC *lcab@ufmg.br

Keywords: $\gamma$-lactams, $\gamma$-lactones, biofilm

\section{INTRODUCTION}

Biofilms are a bacterial communities enclosed within an extracellular matrix capable to survive to antibiotics treatments ${ }^{1}$. It has been estimated that $65-80 \%$ of the microbial infections occurring in the human body are biofilm-related. Therefore, identifying substances that are able to inhibit biofilm formation constitutes a promising approach for the development of new drugs.

In this work, analogues to rubrolides, a class of natural $\gamma$-alkylidene- $\gamma$-lactones ${ }^{3}$, and their corresponding lactams were synthesized and evaluated against $E$. faecalis biofilm formation.

\section{RESULTS AND DISCUSSION}

A sequence of reactions consisting of reduction, Suzuki cross-coupling and alkylidenation allowed the preparation of rubrolides analogues 7-13 (Scheme 1).
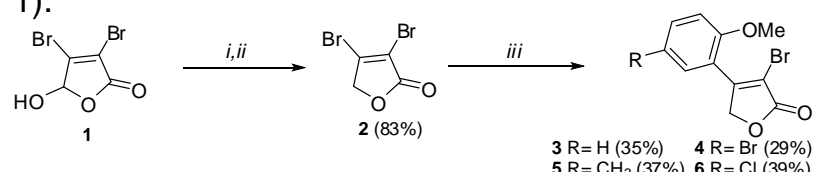

$\begin{array}{lll}5 \mathrm{R}=\mathrm{H}(35 \%) & 4 \mathrm{R}=\mathrm{Br}(29 \%) \\ \mathrm{R}=\mathrm{CH}_{3}(37 \%) & \mathbf{R}=\mathrm{Cl}(39 \%)\end{array}$
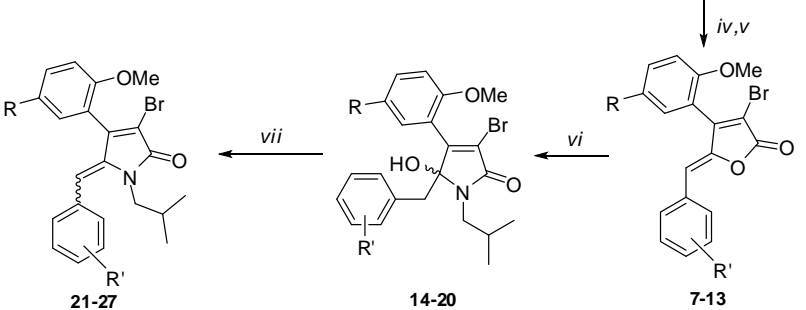

14-20

7-13

i) 1,5 equiv. $\mathrm{NaBH}_{4}, \mathrm{MeOH}, 0^{\circ} \mathrm{C}$; ii) $\mathrm{H}_{2} \mathrm{SO}_{4}$ conc., $\mathrm{MeOH}, 0{ }^{\circ} \mathrm{C}$; iii) ácido borônico, $\mathrm{AsPh}_{3}, \mathrm{PdCl}_{2}(\mathrm{MeCN})_{2}, \mathrm{Ag}_{2} \mathrm{O}, \mathrm{THF}, 65^{\circ} \mathrm{C}, 24 \mathrm{~h}$; iv) aldeído, TBDMSOTf, DIPEA, DCM; v) $\mathrm{DBU}$, refluxo; vi) isopropilamina, $\mathrm{DCM}, 0^{\circ} \mathrm{C}$, $3 \mathrm{~h}$; vii) $p-\mathrm{TsOH}, \mathrm{CHCl}_{3}$, refluxo, $2 \mathrm{~h}$.

\begin{tabular}{|c|c|c|c|c|c|}
\hline$R$ & $\mathrm{R}^{\prime}$ & Comp* (\%) & Comp* $(\%)$ & Comp $^{*}(\%)$ & Comp $^{*}(\%)$ \\
\hline $\mathrm{H}$ & $p-\mathrm{CF}_{3}$ & $7(63)$ & $14(85)$ & $21 Z(36)$ & 21E (39) \\
\hline $\mathrm{Br}$ & $m-\mathrm{Cl}$ & $8(44)$ & $15(78)$ & $22 Z(28)$ & 22E (31) \\
\hline $\mathrm{Br}$ & $p$-Br & $9(45)$ & $16(85)$ & $23 Z(23)$ & 23E (33) \\
\hline $\mathrm{CH}_{3}$ & $m-\mathrm{Cl}$ & $10(63)$ & $17(84)$ & $24 Z(35)$ & 24E (45) \\
\hline $\mathrm{CH}_{3}$ & $0-\mathrm{Cl}$ & $11(68)$ & $18(84)$ & $25 Z(29)$ & 25E (39) \\
\hline $\mathrm{Cl}$ & $o-\mathrm{Br}$ & $12(58)$ & $19(76)$ & $26 Z(29)$ & 26E (43) \\
\hline $\mathrm{Cl}$ & $m-\mathrm{OCH}_{3}$ & $13(65)$ & $20(76)$ & $27 Z(41)$ & 27E (35) \\
\hline
\end{tabular}

Scheme 1. Synthesis of the compounds
These analogues were treated with isobutylamine to prepare the $\gamma$-hydroxy- $\gamma$-lactams $\mathbf{1 4 - 2 0}$ in yields ranging from $76 \%$ to $85 \%$. These compounds were further dehydrated to generate $\gamma$-alkylidene- $\gamma$ lactams 21-27 as mixture of isomers $Z$ and $E$ that were purified by column chromatography (Scheme 1). The configuration of the exocyclic $\mathrm{C} 5-\mathrm{C} 6$ double bound was secured by NOE difference spectroscopy studies.

The lactones and lactams were evaluated against Enterococcus faecalis biofilm formation. Table 1 present the concentration of each compound necessary to inhibit $50 \%$ of biofilm formation $\left(\mathrm{IC}_{50}\right)$.

The most active groups of compounds were the $Z$ and $E \gamma$-alkylidene- $\gamma$-lactams.

Table 1. Effect of the compounds against E. faecalis biofilm formation

\begin{tabular}{cccccccc}
\hline Comp & $\mathrm{IC}_{50}{ }^{*}$ & Comp & $\mathrm{IC}_{50}{ }^{*}$ & Comp & $\mathrm{IC}_{50}{ }^{*}$ & Comp & $\mathrm{IC}_{50}{ }^{*}$ \\
\hline $\mathbf{7}$ & ${ }^{*}$ & $\mathbf{1 4}$ & ${ }^{*}$ & $\mathbf{2 1 Z}$ & $12,0 \pm 4,6$ & $\mathbf{2 1 E}$ & $3,0 \pm 0,7$ \\
$\mathbf{8}$ & $6,9 \pm 1,7$ & $\mathbf{1 5}$ & $>87,5$ & $\mathbf{2 2 Z}$ & $3,3 \pm 1,3$ & $\mathbf{2 2 E}$ & $3,5 \pm 0,2$ \\
$\mathbf{9}$ & $18,7 \pm 5,1$ & $\mathbf{1 6}$ & $33,4 \pm 17,0$ & $\mathbf{2 3 Z}$ & $6,6 \pm 0,4$ & $\mathbf{2 3 E}$ & $3,4 \pm 0,4$ \\
$\mathbf{1 0}$ & $1,5 \pm 0,1$ & $\mathbf{1 7}$ & $>87,5$ & $\mathbf{2 4 Z}$ & $62,6 \pm 9,7$ & $\mathbf{2 4 E}$ & $59,3 \pm 11,1$ \\
$\mathbf{1 1}$ & $*$ & $\mathbf{1 8}$ & $1,1 \pm 0,1$ & $\mathbf{2 5 Z}$ & $1,1 \pm 0,3$ & $\mathbf{2 5 E}$ & $1,0 \pm 0,2$ \\
$\mathbf{1 2}$ & $>87,5$ & $\mathbf{1 9}$ & $1,3 \pm 0,2$ & $\mathbf{2 6 Z}$ & $1,5 \pm 0,1$ & $\mathbf{2 6 E}$ & $0,76 \pm 0,2$ \\
$\mathbf{1 3}$ & $53,1 \pm 16,7$ & $\mathbf{2 0}$ & $>87,5$ & $\mathbf{2 7 Z}$ & $1,5 \pm 0,3$ & $\mathbf{2 7 E}$ & $3,3 \pm 1,5$ \\
$*$ & \multicolumn{7}{|c}{ values expressed in $\mu \mathrm{g} / \mathrm{mL}}$.
\end{tabular}

\section{CONCLUSION}

Seven analogues to rubrolides were prepared and then converted into their corresponding lactams through the two-step lactamization. The evaluation of these compounds against $E$. faecalis biofilm formation showed that all these groups of compounds are very active, with the $\gamma$-alkylidene- $\gamma$ lactams amongst the most actives.

\section{ACKNOWLEDGEMENTS}

CAPES, UFV, CNPq, FAPEMIG

\section{REFERENCES}

${ }^{1}$ Musk Jr, D. J.; Hergenrother, P. J. Curr. Med. Chem., 2006, 13, 2163.

${ }^{2}$ Davies, D. Nat. Rev. Drug Discov., 2010, 2, 114

${ }^{3}$ Miao, S.; Andersen, R. J. J. Org. Chem., 56, 1991, 6275. 